\title{
An inventory model for perishable items with quadratic trapezoidal type demand under partial backlogging
}

\author{
Smrutirekha Debata ${ }^{a}$, Milu Acharya ${ }^{b}$ and G. C. Samanta ${ }^{*}$
}

\author{
${ }^{a}$ Research Scholar, Department of Mathematics, Utkal University, Bhubaneswar, Odisha, India \\ ${ }^{b}$ Department of Mathematics, SOA University, Bhubaneswar, Odisha, India \\ ${ }^{\mathrm{c}}$ Department of Mathematics, Birla Institute of Technology and Science (BITS) Pilani, Goa Campus, Goa, India
}

\section{CHRONICLE ABSTRACT}

\section{Article history:}

Received March 92014

Received in Revised Format

August 52014

Accepted December 32014

Available online

December 32014

Keywords:

Quadratic trapezoidal demand

Deterioration

Shortages

Partial backlogging
In this paper, we consider the inventory model for perishable items with quadratic trapezoidal type demand rate, that is, the demand rate is a piecewise quadratic function under constant deterioration rate. The model consider allows for shortages and the demand is partially backlogged. The model is solved analytically by minimizing the total inventory cost. The result is illustrated with numerical example. Finally, we discuss sensitivity analysis for the model.

\section{Introduction}

Deteriorating items are very common issue in our daily life circumstances. In recent years, many researchers have studied inventory models for deteriorating items, however, academia has not reached a consensus on the definition of the deteriorating items. According to Wee (1993), deteriorating items refers to the items that become decayed, damaged, evaporative, expired, invalid, devaluation and so on through time. According to the definition, deteriorating items can be classified into two categories. The first category refers to the items that become decayed, damaged, evaporative, or expired through time, like meat, vegetables, fruit, medicine, flowers and so on; the other category refers to the items that lose part or total value through time because of new technology or the introduction of alternatives, like computer chips, mobile phones, fashion and seasonal goods and so on. The inventory problem of deteriorating items was first studied by Whitin (1957), he studied fashion items deteriorating at the end of the storage period. Then, Ghare and Schrader (1963) concluded in their study that the consumption of the deteriorating items was closely relative to a negative exponential function of time. Various authors

\footnotetext{
* Corresponding author.

E-mail: gauranga81@gmail.com (G. C. Samanta)

(C) 2014 Growing Science Ltd. All rights reserved. doi: $10.5267 / j$. .jiec.2014.12.001
} 
such as Deng et al. (2007), Cheng and Wang (2009), Cheng et al. (2011) and Hung (2011) studied inventory models for deteriorating items in various aspects.

In world business market, demand has been always one of the most key factors in the decisions relating to the inventory and production activities. There are mainly two categories demands in the present studies, one is deterministic demand and the other is stochastic demand. Various formations of consumption tendency have been studied, such as constant demand (Padmanabhan \& Vrat, 1990; Chung \& Lin, 2001; Benkherouf et al., 2003; Chu et al., 2004), level-dependent demand (Giri \& Choudhuri, 1998; Chung et al., 2000; Bhattacharya, 2005; Wu et al., 2006), price dependent demand (Wee \& Law, 1999; Abad, 1996, 2001), time dependent demand (Resh et al., 1976; Henery, 1979; Sachan, 1984; Dave, 1989; Teng, 1996; Teng et al., 2002; Skouri \& Papachristos, 2002; Panda et al., 2012; Sett et al., 2013; Mishra et al., 2013) and time and price dependent demand (Wee, 1995). Among them, ramp type demand is a special type of time dependent demand. Hill (1995), one of the pioneers, developed an inventory model with ramp type demand that begins with a linear increasing demand until to the turning point, denoted as $\mu$, proposed by previous researchers, then it becomes a constant demand. There has been a movement towards developing this type of inventory system for minimum cost and maximum profit problems. Several authors: Mandal and Pal (1998) focused on deteriorating items. Wu et al. (1999) were concerned with backlog rates relative to the waiting time. Wu and Ouyang (2000) tried to build an inventory system under two replenishment policies: starting with shortage or without shortage. Wu (2001) considered the deteriorated items satisfying Weibull distribution. Giri et al (2003) dealt with more generalized three parameter Weibull deterioration distribution. Deng (2005) extended the inventory model of $\mathrm{Wu}$ et al. (1999) for the situation where the in-stock period is shorter than $\mu$. Manna and Chaudhuri (2006) set up a model where the deterioration is dependent on time. Panda et al. (2007) constructed an inventory model with a comprehensive ramp type demand. Deng et al. (2007) contributed to the revision of Mandal and Pal (1998), and Wu and Ouyang (2000). Panda et al. (2008) examined the cyclic deterioration items. Wu et al. (2008) studied the maximum profit problem with the stockdependent selling rate. They developed two inventory models all related to the conversion of the ramp type demand, and then examined the optimal solution for each case. However, in a realistic product life cycle, demand is increasing with time during the growth phase. Then, after reaching its peak, the demand becomes stable for a finite time period called the maturity phase. Thereafter, the demand starts decreasing with time and eventually reaching zero or constant.

In this work, we extend Hill's ramp type demand rate to quadratic trapezoidal type demand rate. Such type of demand pattern is generally seen in the case of any fad or seasonal goods coming to market. The demand rate for such items increases quadratic-ally with the time up to certain time and then ultimately stabilizes and becomes constant, and finally the demand rate approximately decreases to a constant, and then begins the next replenishment cycle. We think that such type of demand rate is quite natural and useful in real world market situation. One can think that our work may provide a solid foundation for the future study of this kind of important inventory models with quadratic trapezoidal type demand rate.

\section{Assumption and notations}

The fundamental assumption and notations used in this paper are given as follows:

(1)The demand rate, $R(t)$, which is positive and consecutive, is assumed to be a quadratic trapezoidal type function of time, that is

$$
R(t)= \begin{cases}a_{1}+b_{1} t+c_{1} t^{2}, & t \leq \mu_{1}, \\ R_{0}, & \mu_{1} \leq t \leq \mu_{2}, \\ a_{2}+b_{2} t-c_{2} t^{2}, & \mu_{2} \leq t \leq T\end{cases}
$$


Chose $a_{1}, b_{1}, c_{1}, a_{2}, b_{2}$ and $c_{2}$ such a way that $a_{2}+b_{2} t-c_{2} t^{2}$ should not be negative for $\mu_{2} \leq t \leq T$. where $\mu_{1}$ is the time point changing from the increasing quadratic demand to constant demand, and $\mu_{2}$ is the time point changing from the constant demand to the decreasing demand.

(2) Replenishment rate is infinite, thus replenishment is instantaneous.

(3) $I(t)$ is the inventory level at any time $t, 0 \leq t \leq T$.

(4) $T$ is the fixed length of each ordering cycle.

(5) $\theta$ is the constant rate of deterioration, $0<\theta<1$.

(6) $t_{1}$ is the time when the inventory level reaches zero.

(7) $t_{1}{ }^{*}$ is an optimal point.

(8) $k_{0}$ is the fixed ordering cost per order.

(9) $k_{1}$ is the cost of each deteriorated item.

(10) $k_{2}$ is the inventory holding cost per unit per unit of time.

(11) $k_{3}$ is the shortage cost per unit per unit of time.

(12) $S$ is the maximum inventory level for the ordering cycle, such that $S=I(0)$.

(13) $Q$ is the ordering quantity per cycle.

(14) $A_{1}\left(t_{1}\right)$ is the average total cost per unit time under the condition $t_{1} \leq \mu_{1}$.

(15) $A_{2}\left(t_{1}\right)$ is the average total cost per unit time, for $\mu_{1} \leq t_{1} \leq \mu_{2}$.

(16) $A_{3}\left(t_{1}\right)$ is the average total cost per unit time, for $\mu_{2} \leq t_{1} \leq T$.

\section{Mathematical and theoretical results}

Here, we consider the deteriorating inventory model where demand rate is trapezoidal type quadratic function. Replenishment occurs at time $t=0$ when the inventory level attains its maximum. For $t \in\left[0, t_{1}\right]$ , the inventory level reduces due to both demand and deterioration. At time $t_{1}$, the inventory level reaches zero, then shortage is allowed to occur during the interval $\left(t_{1}, T\right)$, and all of the demand during the shortage period $\left(t_{1}, T\right)$ is completely backlogged. The total amount of backlogged items is replaced by the next replenishment. The rate of change of the inventory during the stock period $\left[0, t_{1}\right]$ and shortage period $\left(t_{1}, T\right)$ is governed by the following differential equations:

$$
\begin{aligned}
& \frac{d I(t)}{d t}+\theta I(t)+R(t)=0,0<t<t_{1}, \\
& \frac{d I(t)}{d t}+R(t)=0, t_{1}<t<T,
\end{aligned}
$$

with boundary condition $I(0)=S$ and $I\left(t_{1}\right)=0$. One can think about $t_{1}, t_{1}$ may occur within $\left[0, \mu_{1}\right]$ or $\left[\mu_{1}, \mu_{2}\right]$ or $\left[\mu_{2}, T\right]$. Hence in this paper we are going to discuss all three possible cases.

Case 1: $0<t_{1} \leq \mu_{1}$

The quadratic trapezoidal type market demand and constant rate of deterioration, the inventory level gradually diminishes during the period $\left[0, t_{1}\right]$ and ultimately reaches to zero at time $t=t_{1}$. Then, from Eq. (2) and Eq. (3), we have

$$
\begin{aligned}
& \frac{d I(t)}{d t}+\theta I(t)+a_{1}+b_{1} t+c_{1} t^{2}=0,0<t<t_{1} \\
& \frac{d I(t)}{d t}+a_{1}+b_{1} t+c_{1} t^{2}=0, t_{1}<t<\mu_{1}
\end{aligned}
$$




$$
\begin{aligned}
& \frac{d I(t)}{d t}+R_{0}=0, \quad \mu_{1}<t<\mu_{2} \\
& \frac{d I(t)}{d t}+a_{2}+b_{2} t-c_{2} t^{2}=0, \quad \mu_{2}<t<T
\end{aligned}
$$

Now solving the differential Eqs. (4-7) with the condition $I\left(t_{1}\right)=0$ and continuous property of $I(t)$, we get

$$
\begin{aligned}
& I(t)=\left(\frac{a_{1}+b_{1} t_{1}+c_{1} t_{1}^{2}}{\theta}-\frac{b_{1}+2 c_{1} t_{1}}{\theta^{2}}+\frac{2 c_{1}}{\theta^{3}}\right) e^{\theta\left(t_{1}-t\right)}-\frac{a_{1}+b_{1} t+c_{1} t^{2}}{\theta}+\frac{b_{1}+2 c_{1} t}{\theta^{2}}-\frac{2 c_{1}}{\theta^{3}}, 0 \leq t \leq t_{1} \\
& I(t)=\left(t_{1}-t\right) a_{1}+\left(t_{1}^{2}-t^{2}\right) \frac{b_{1}}{2}+\left(t_{1}^{3}-t^{3}\right) \frac{c_{1}}{3}, t_{1} \leq t \leq \mu_{1} \\
& I(t)=-R_{0} t+a_{1} t_{1}+\left(t_{1}^{2}+\mu_{1}^{2}\right) \frac{b_{1}}{2}+\left(t_{1}^{3}+2 \mu_{1}^{3}\right) \frac{c_{1}}{3}, \mu_{1} \leq t \leq \mu_{2} \\
& I(t)=a_{1} t_{1}-a_{2} t+\left(t_{1}^{2}+\mu_{1}^{2}\right) \frac{b_{1}}{2}+\left(t_{1}^{3}+2 \mu_{1}^{3}\right) \frac{c_{1}}{3}-\left(t^{2}+\mu_{2}^{2}\right) \frac{b_{2}}{2}+\left(t^{3}+2 \mu_{2}^{3}\right) \frac{c_{2}}{3}, \mu_{2} \leq t \leq T
\end{aligned}
$$

The beginning inventory level can be computed as

$$
S=I(0)=\left(\frac{a_{1}}{\theta}-\frac{b_{1}}{\theta^{2}}+\frac{2 c_{1}}{\theta^{3}}\right)\left(e^{\theta_{1}}-1\right)+\left(\frac{b_{1} t_{1}+c_{1} t_{1}^{2}}{\theta}-\frac{2 c_{1} t_{1}}{\theta^{2}}\right) e^{\theta_{1}}
$$

The total number of items which is perish in the interval $\left[0, t_{1}\right]$, say $D_{T}$, is

$$
\begin{aligned}
D_{T}= & S-\int_{0}^{t_{1}} R(t) d t=S-\int_{0}^{t_{1}}\left(a_{1}+b_{1} t+c_{1} t^{2}\right) d t \\
& =\left(\frac{a_{1}}{\theta}-\frac{b_{1}}{\theta^{2}}+\frac{2 c_{1}}{\theta^{3}}\right)\left(e^{\theta_{1}}-1\right)+\left(\frac{b_{1} t_{1}+c_{1} t_{1}^{2}}{\theta}-\frac{2 c_{1} t_{1}}{\theta^{2}}\right) e^{\theta_{1}}-a_{1} t_{1}-\frac{b_{1} t_{1}^{2}}{2}-\frac{c_{1} t_{1}^{3}}{3}
\end{aligned}
$$

The total amounts of inventory carried during the interval $\left[0, t_{1}\right]$, say $C_{T}$, is

$$
\begin{aligned}
C_{T}=\int_{0}^{t_{1}} I(t) d t & =\int_{0}^{t_{1}}\left[\begin{array}{l}
\left(\frac{a_{1}+b_{1} t_{1}+c_{1} t_{1}^{2}}{\theta}-\frac{b_{1}+2 c_{1} t_{1}}{\theta^{2}}+\frac{2 c_{1}}{\theta^{3}}\right) e^{\theta\left(t_{1}-t\right)} \\
-\frac{a_{1}+b_{1} t+c_{1} t^{2}}{\theta}+\frac{b_{1}+2 c_{1} t}{\theta^{2}}-\frac{2 c_{1}}{\theta^{3}}
\end{array}\right] d t \\
& =\left(\frac{a_{1}+b_{1} t_{1}+c_{1} t_{1}^{2}}{\theta^{2}}-\frac{b_{1}+2 c_{1} t_{1}}{\theta^{3}}+\frac{2 c_{1}}{\theta^{4}}\right)\left(e^{\theta t_{1}}-1\right)+\left(\frac{b_{1}}{\theta^{2}}-\frac{a_{1}}{\theta}-\frac{2 c_{1}}{\theta^{3}}\right) t_{1}+\left(\frac{c_{1}}{\theta^{2}}-\frac{b_{1}}{2 \theta}\right) t_{1}^{2}-\frac{c_{1}}{3 \theta} t_{1}^{3}
\end{aligned}
$$

The total shortage quantity during the interval $\left[\mathrm{t}_{1}, \mathrm{~T}\right]$, say $\mathrm{B}_{\mathrm{T}}$, is

$$
\begin{aligned}
B_{T}= & -\int_{t_{1}}^{T} I(t) d t=-\int_{t_{1}}^{\mu_{1}} I(t) d t-\int_{\mu_{1}}^{\mu_{2}} I(t) d t-\int_{\mu_{2}}^{T} I(t) d t=-\int_{t_{1}}^{\mu_{1}}\left[\left(t_{1}-t\right) a_{1}+\left(t_{1}^{2}-t^{2}\right) \frac{b_{1}}{2}+\left(t_{1}^{3}-t^{3}\right) \frac{c_{1}}{3}\right] d t \\
& -\int_{\mu_{1}}^{\mu_{2}}\left[-R_{0} t+a_{1} t_{1}+\left(t_{1}^{2}+\mu_{1}^{2}\right) \frac{b_{1}}{2}+\left(t_{1}^{3}+2 \mu_{1}^{3}\right) \frac{c_{1}}{3}\right] d t
\end{aligned}
$$




$$
\begin{gathered}
-\int_{\mu_{2}}^{T}\left[a_{1} t_{1}-a_{2} t+\left(t_{1}^{2}+\mu_{1}^{2}\right) \frac{b_{1}}{2}+\left(t_{1}^{3}+2 \mu_{1}^{3}\right) \frac{c_{1}}{3}-\left(t^{2}+\mu_{2}^{2}\right) \frac{b_{2}}{2}+\left(t^{3}+2 \mu_{2}^{3}\right) \frac{c_{2}}{3}\right] d t \\
=-\left(t_{1} \mu_{1}-t_{1}^{2}\right) a_{1}+\frac{a_{1}}{2}\left(\mu_{1}^{2}-t_{1}^{2}\right)-\frac{b_{1}}{2} t_{1}^{2}\left(\mu_{1}-t_{1}\right)+\frac{b_{1}}{6}\left(\mu_{1}^{3}-t_{1}^{3}\right)-\frac{c_{1}}{3} t_{1}^{3}\left(\mu_{1}-t_{1}\right)+\frac{c_{1}}{12}\left(\mu_{1}^{4}-t_{1}^{4}\right) \\
+\frac{R_{0}}{2}\left(\mu_{1}^{2}-\mu_{2}^{2}\right)-a_{1} t_{1}\left(\mu_{2}-\mu_{1}\right)-\frac{b_{1}}{2} t_{1}^{2}\left(\mu_{2}-\mu_{1}\right)-\frac{b_{1}}{2} \mu_{1}^{2}\left(\mu_{2}-\mu_{1}\right)-\frac{c_{1}}{3} t_{1}^{3}\left(\mu_{2}-\mu_{1}\right) \\
-\frac{2 c_{1}}{3} \mu_{1}^{3}\left(\mu_{2}-\mu_{1}\right)-a_{1} t_{1}\left(T-\mu_{2}\right)+\frac{a_{2}}{2}\left(T^{2}-\mu_{2}^{2}\right)-\frac{b_{1}}{2} t_{1}^{2}\left(T-\mu_{2}\right)-\frac{b_{1}}{2} \mu_{1}^{2}\left(T-\mu_{2}\right) \\
-\frac{c_{1}}{3}\left(t_{1}^{3}+2 \mu_{1}^{3}\right)\left(T-\mu_{2}\right)+\frac{b_{2}}{6}\left(T^{3}-\mu_{2}^{3}\right)+\frac{b_{2}}{2} \mu_{2}^{2}\left(T-\mu_{2}\right)-\frac{c_{2}}{12}\left(T^{4}-\mu_{2}^{4}\right)-\frac{2 c_{2}}{3} \mu_{2}^{3}\left(T-\mu_{2}\right)
\end{gathered}
$$

The average total cost per unit time for $0<t_{1} \leq \mu_{1}$ is given by

$$
A_{1}\left(t_{1}\right)=\frac{1}{T}\left[k_{0}+k_{1} D_{T}+k_{2} C_{T}+k_{3} B_{T}\right]
$$

The first order derivative of $A_{1}\left(t_{1}\right)$ with respect to $t_{1}$ is as follows:

$$
\frac{d A_{1}\left(t_{1}\right)}{d t_{1}}=\frac{1}{T}\left[\left(k_{1}+\frac{k_{2}}{\theta}\right)\left(e^{\theta_{1}}-1\right)+k_{3}\left(t_{1}-T\right)\right]\left(a_{1}+b_{1} t_{1}+c_{1} t_{1}^{2}\right)
$$

The necessary condition for $A_{1}\left(t_{1}\right)$ to be minimized, is $\frac{d A_{1}\left(t_{1}\right)}{d t_{1}}=0$, that is

$$
\frac{1}{T}\left[\left(k_{1}+\frac{k_{2}}{\theta}\right)\left(e^{\theta_{1}}-1\right)+k_{3}\left(t_{1}-T\right)\right]\left(a_{1}+b_{1} t_{1}+c_{1} t_{1}^{2}\right)=0
$$

This implies that

$$
\begin{aligned}
& {\left[\left(k_{1}+\frac{k_{2}}{\theta}\right)\left(e^{\theta_{1}}-1\right)+k_{3}\left(t_{1}-T\right)\right]=0} \\
& \text { Let } p\left(t_{1}\right)=\left(k_{1}+\frac{k_{2}}{\theta}\right)\left(e^{\theta_{1}}-1\right)+k_{3}\left(t_{1}-T\right),
\end{aligned}
$$

Since $p(0)=-k_{3} T<0, p(T)=\left(k_{1}+\frac{k_{2}}{\theta}\right)\left(e^{\theta T}-1\right)>0$ and $p^{\prime}\left(t_{1}\right)=\left(k_{1}+\frac{k_{1}}{\theta}\right) e^{\theta_{1}} \theta+k_{3}>0$, it implies that $p\left(t_{1}\right)$ is a strictly monotonically increasing function and Eq. (19) has a unique solution at $t_{1}^{*}$, for $t_{1}^{*} \in(0, T)$. Therefore, we have

\section{Property-1}

The constant deteriorating rate of an inventory model with quadratic trapezoidal type demand rate under the time interval $0<t_{1} \leq \mu_{1}, A_{1}\left(t_{1}\right)$ attains its minimum at $t_{1}=t_{1}^{*}$, where $p\left(t_{1}^{*}\right)=0$ if $t_{1}^{*}<\mu_{1}$. On the other hand, $A_{1}\left(t_{1}\right)$ attains its minimum at $t_{1}^{*}=\mu_{1}$ if $t_{1}^{*} \geq \mu_{1}$. 
The total back order amount at the end of the cycle is follows,

$$
\Delta_{1}=-a_{1} t_{1}^{*}+a_{2} T-\frac{b_{1}}{2}\left(t_{1}^{* 2}+\mu_{1}^{2}\right)-\frac{c_{1}}{3}\left(t_{1}^{* 3}+2 \mu_{1}^{3}\right)+\frac{b_{2}}{2}\left(T^{2}+\mu_{2}^{2}\right)-\frac{c_{2}}{3}\left(T^{3}+2 \mu_{2}^{3}\right)
$$

Therefore, the optimal order quantity, denoted by $Q^{*}$, is $Q^{*}=S^{*}+\Delta_{1}$, where $S^{*}$ denote the optimal value of $S$.

Case-II, $\mu_{1} \leq t_{1} \leq \mu_{2}$

For the time period $t_{1} \in\left[\mu_{1}, \mu_{2}\right]$, then, the differential equations governing the inventory model can be expressed as follows:

$$
\begin{aligned}
& \frac{d I(t)}{d t}+\theta I(t)+a_{1}+b_{1} t+c_{1} t^{2}=0,0<t<\mu_{1} \\
& \frac{d I(t)}{d t}+\theta I(t)+R_{0}=0, \mu_{1}<t<t_{1} \\
& \frac{d I(t)}{d t}+R_{0}=0, \quad t_{1}<t<\mu_{2} \\
& \frac{d I(t)}{d t}+a_{2}+b_{2} t-c_{2} t^{2}=0, \mu_{2}<t<T
\end{aligned}
$$

Solving differential Eq. (22-25), using $I\left(t_{1}\right)=0$, we get

$$
\begin{aligned}
& I(t)=\left(\frac{R_{0}}{\theta} e^{\theta t_{1}}-\frac{b_{1}}{\theta^{2}} e^{\theta \mu_{1}}\right) e^{-\theta t}-\frac{a_{1}+b_{1} t+c_{1} t^{2}}{\theta}+\frac{b_{1}+2 c_{1} t}{\theta^{2}}-\frac{2 c_{1}}{\theta^{3}}-\frac{2 c_{1} \mu_{1}}{\theta^{2}} e^{\theta\left(\mu_{1}-t\right)}+\frac{2 c_{1}}{\theta^{3}} e^{\theta\left(\mu_{1}-t\right)}, 0 \leq t \leq \mu_{1} \\
& I(t)=\frac{R_{0}}{\theta}\left(e^{\theta\left(t_{1}-t\right)}-1\right), \quad \mu_{1} \leq t \leq t_{1} \\
& I(t)=R_{0}\left(t_{1}-t\right), \quad t_{1} \leq t \leq \mu_{2} \\
& I(t)=R_{0} t_{1}-a_{2} t-\frac{b_{2}}{2}\left(t^{2}+\mu_{2}^{2}\right)+\frac{c_{2}}{3}\left(t^{3}+2 \mu_{2}^{3}\right), \mu_{2} \leq t \leq T
\end{aligned}
$$

The beginning inventory can be computed as

$$
S=I(0)=\frac{R_{0}}{\theta} e^{\theta \epsilon_{1}}-\frac{b_{1}}{\theta^{2}} e^{\theta \mu_{1}}-\frac{a_{1}}{\theta}+\frac{b_{1}}{\theta^{2}}-\frac{2 c_{1}}{\theta^{3}}-\frac{2 c_{1} \mu_{1}}{\theta^{2}} e^{\theta \mu_{1}}+\frac{2 c_{1}}{\theta^{3}} e^{\theta \mu_{1}}
$$

The total amount of items which is perish within the time interval $\left[0, t_{1}\right]$ is

$$
\begin{aligned}
D_{T} & =S-\int_{0}^{t_{1}} R(t) d t=S-\int_{0}^{\mu_{1}}\left(a_{1}+b_{1} t+c_{1} t^{2}\right) d t-\int_{\mu_{1}}^{t_{1}} R_{0} d t=\frac{R_{0}}{\theta} e^{\theta t_{1}}-\left(\frac{b_{1}}{\theta^{2}}+\frac{2 c_{1} \mu_{1}}{\theta^{2}}-\frac{2 c_{1}}{\theta^{3}}\right) e^{\theta \mu_{1}} \\
& -R_{0}\left(t_{1}-\mu_{1}\right)-a_{1}\left(\mu_{1}+\frac{1}{\theta}\right)+b_{1}\left(\frac{1}{\theta^{2}}-\frac{\mu_{1}^{2}}{2}\right)-c_{1}\left(\frac{2}{\theta^{3}}+\frac{\mu_{1}^{3}}{3}\right)
\end{aligned}
$$

The total amount of inventory carried during the time interval $\left[0, t_{1}\right]$ is 


$$
\begin{aligned}
& C_{T}=\int_{0}^{t_{1}} I(t) d t=\int_{0}^{\mu_{1}} I(t) d t+\int_{\mu_{1}}^{t_{1}} I(t) d t \\
& =\int_{0}^{\mu_{1}}\left[\begin{array}{l}
\left(\frac{R_{0}}{\theta} e^{\theta t_{1}}-\frac{b_{1}}{\theta^{2}} e^{\theta \mu_{1}}\right) e^{-\theta t}-\frac{a_{1}+b_{1} t+c_{1} t^{2}}{\theta}+\frac{b_{1}+2 c_{1} t}{\theta^{2}}-\frac{2 c_{1}}{\theta^{3}} \\
-\frac{2 c_{1} \mu_{1}}{\theta^{2}} e^{\theta\left(\mu_{1}-t\right)}+\frac{2 c_{1}}{\theta^{3}} e^{\theta\left(\mu_{1}-t\right)}
\end{array}\right] d t+\int_{\mu_{1}}^{t_{1}}\left[\frac{R_{0}}{\theta}\left(e^{\theta\left(t_{1}-t\right)}-1\right)\right] d t \\
& =\frac{b_{1}}{\theta^{3}}+\frac{R_{0}}{\theta^{2}} e^{\theta_{1}}-\frac{b_{1}}{\theta^{3}} e^{\theta \mu_{1}}-\frac{1}{\theta}\left(\theta \mu_{1}+\frac{b_{1} \mu_{1}^{2}}{2}+\frac{c_{1} \mu_{1}^{3}}{3}\right)+\frac{b_{1} \mu_{1}}{\theta^{2}}-\frac{2 c_{1} \mu_{1}}{\theta^{3}} e^{\theta \mu_{1}} \\
& -\frac{2 c_{1}}{\theta^{4}}+\frac{2 c_{1}}{\theta^{4}} e^{\theta \mu_{1}}-\frac{R_{0}}{\theta^{2}}-\frac{R_{0}}{\theta}\left(t_{1}-\mu_{1}\right)
\end{aligned}
$$

The total amount of shortage during the interval $\left[t_{1}, T\right]$

$$
\begin{aligned}
B_{T}= & -\int_{t_{1}}^{T} I(t) d t=-\int_{t_{1}}^{\mu_{2}} I(t) d t-\int_{\mu_{2}}^{T} I(t) d t \\
& =-\int_{t_{1}}^{\mu_{2}} R_{0}\left(t_{1}-t\right) d t-\int_{\mu_{2}}^{T}\left[R_{0} t_{1}-a_{2} t-\frac{b_{2}}{2}\left(t^{2}+\mu_{2}^{2}\right)+\frac{c_{2}}{3}\left(t^{3}+2 \mu_{2}^{3}\right)\right] d t \\
& =-R_{0} t_{1}\left(\mu_{2}-t_{1}\right)+\frac{R_{0}}{2}\left(\mu_{2}{ }^{2}-t_{1}{ }^{2}\right)-R_{0} t_{1}\left(T-\mu_{2}\right)+\frac{a_{2}}{2}\left(T^{2}-\mu_{2}{ }^{2}\right)+\frac{b_{2}}{6}\left(T^{3}-\mu_{2}{ }^{3}\right) \\
& +\frac{b_{2}}{2} \mu_{2}^{2}\left(T-\mu_{2}\right)-\frac{c_{2}}{12}\left(T^{4}-\mu_{2}{ }^{4}\right)-\frac{2 c_{2}}{3} \mu_{2}^{3}\left(T-\mu_{2}\right)
\end{aligned}
$$

Now, the average total cost per unit time under the condition $\mu_{1} \leq t_{1} \leq \mu_{2}$, can be obtained as

$$
A_{2}\left(t_{1}\right)=\frac{1}{T}\left[k_{0}+k_{1} D_{T}+k_{2} C_{T}+k_{3} B_{T}\right]
$$

The first order derivative of $A_{2}\left(t_{1}\right)$ with respect to $t_{1}$ is given by

$$
\frac{d A_{2}\left(t_{1}\right)}{d t_{1}}=\frac{R_{0}}{T}\left[\left(k_{1}+\frac{k_{2}}{\theta}\right)\left(e^{\theta t_{1}}-1\right)+k_{3}\left(t_{1}-T\right)\right]
$$

The required necessary condition for $A_{2}\left(t_{1}\right)$ to be minimized is $\frac{d A_{2}\left(t_{1}\right)}{d t_{1}}=0$, that is

$$
\begin{aligned}
& {\left[\left(k_{1}+\frac{k_{2}}{\theta}\right)\left(e^{\theta_{1}}-1\right)+k_{3}\left(t_{1}-T\right)\right]=0} \\
& \text { Let } p\left(t_{1}\right)=\left(k_{1}+\frac{k_{2}}{\theta}\right)\left(e^{\theta_{1}}-1\right)+k_{3}\left(t_{1}-T\right),
\end{aligned}
$$

since $p^{\prime}\left(t_{1}\right)=\left(k_{1}+\frac{k_{1}}{\theta}\right) e^{a_{1}} \theta+k_{3}>0$, which implies that $p\left(t_{1}\right)$ is strictly monotonically increasing function during the interval $\mu_{1} \leq t_{1} \leq \mu_{2}$.

\section{Property-2}

The constant deteriorating rate of an inventory model with quadratic trapezoidal type demand function during the time interval $\mu_{1} \leq t_{1} \leq \mu_{2}, A_{2}\left(t_{1}\right)$ attains its minimum at $t_{1}^{*}=\mu_{1}$ if $t_{1}^{*}<\mu_{1}$ and $A_{2}\left(t_{1}\right)$ attains its minimum at $t_{1}^{*}=\mu_{2}$ if $\mu_{2}<t_{1}^{*}$. 
Now, we can calculate the total amount of back-order quantity at the end of the cycle is

$$
\Delta_{2}=-R_{0} t_{1}^{*}+a_{2} T+\frac{b_{2}}{2}\left(T^{2}+\mu_{2}^{2}\right)-\frac{c_{2}}{3}\left(T^{3}+2 \mu_{2}^{3}\right)
$$

Therefore, the optimal order quantity denoted by $Q^{*}$ is $Q^{*}=S^{*}+\Delta_{2}$, where $S^{*}$ denotes the optimal vale of $S$.

\section{Case-III $\mu_{2} \leq t_{1}<T$}

For the time interval $t_{1} \in\left[\mu_{2}, T\right)$, then, the differential equations governing the inventory model can be expressed as follows:

$$
\begin{aligned}
& \frac{d I(t)}{d t}+\theta I(t)+a_{1}+b_{1} t+c_{1} t^{2}=0,0<t<\mu_{1} \\
& \frac{d I(t)}{d t}+\theta I(t)+R_{0}=0, \quad \mu_{1}<t<\mu_{2} \\
& \frac{d I(t)}{d t}+\theta I(t)+a_{2}+b_{2} t-c_{2} t^{2}=0, \quad \mu_{2}<t<t_{1} \\
& \frac{d I(t)}{d t}+a_{2}+b_{2} t-c_{2} t^{2}=0, \quad t_{1}<t<T
\end{aligned}
$$

Solving the differential Eqs. (39-42) with $I\left(t_{1}\right)=0$, we can get

$$
\begin{aligned}
I(t)= & \frac{b_{1}+2 c_{1} t}{\theta^{2}}-\frac{2 c_{1}}{\theta^{3}}-\frac{a_{1}+b_{1} t+c_{1} t^{2}}{\theta}+\left(\frac{a_{2}+b_{2} t_{1}-c_{2} t_{1}^{2}}{\theta}-\frac{b_{2}}{\theta^{2}}+\frac{2 c_{2} t_{1}}{\theta^{2}}-\frac{2 c_{2}}{\theta^{3}}\right) e^{\theta\left(t_{1}-t\right)} \\
& +\left(\frac{b_{2}-2 c_{2} \mu_{2}}{\theta^{2}}+\frac{2 c_{2}}{\theta^{3}}\right) e^{\theta\left(\mu_{2}-t\right)}+\left(\frac{2 c_{1}}{\theta^{3}}-\frac{b_{1}+2 c_{1} \mu_{1}}{\theta^{2}}\right) e^{\theta\left(\mu_{1}-t\right)}, 0 \leq t \leq \mu_{1} \\
I(t)= & -\frac{R_{0}}{\theta}+\left(\frac{a_{2}+b_{2} t_{1}-c_{2} t_{1}^{2}}{\theta}-\frac{b_{2}}{\theta^{2}}+\frac{2 c_{2} t_{1}}{\theta^{2}}-\frac{2 c_{2}}{\theta^{3}}\right) e^{\theta\left(t_{1}-t\right)}+\left(\frac{b_{2}-2 c_{2} \mu_{2}}{\theta^{2}}+\frac{2 c_{2}}{\theta^{3}}\right) e^{\theta\left(\mu_{2}-t\right)} \\
\mu_{1} \leq & t \leq \mu_{2} \\
I(t)= & \left(\frac{a_{2}+b_{2} t_{1}-c_{2} t_{1}^{2}}{\theta}-\frac{b_{2}}{\theta^{2}}+\frac{2 c_{2} t_{1}}{\theta^{2}}-\frac{2 c_{2}}{\theta^{3}}\right) e^{\theta\left(t_{1}-t\right)}+\frac{b_{2}-2 c_{2} t}{\theta^{2}}+\frac{2 c_{2}}{\theta^{3}}-\frac{a_{2}+b_{2} t-c_{2} t^{2}}{\theta}, \\
& \mu_{2} \leq t \leq t_{1} \\
I(t)= & a_{2}\left(t_{1}-t\right)+\frac{b_{2}}{2}\left(t_{1}^{2}-t^{2}\right)+\frac{c_{2}}{3}\left(t^{3}-t_{1}^{3}\right), t_{1} \leq t \leq T
\end{aligned}
$$

The total amount of inventory level at the beginning can be computed as

$$
\begin{gathered}
S=I(0)=\frac{b_{1}}{\theta^{2}}-\frac{2 c_{1}}{\theta^{3}}-\frac{a_{1}}{\theta}+\left(\frac{a_{2}+b_{2} t_{1}-c_{2} t_{1}^{2}}{\theta}-\frac{b_{2}}{\theta^{2}}+\frac{2 c_{2} t_{1}}{\theta^{2}}-\frac{2 c_{2}}{\theta^{3}}\right) e^{\theta t_{1}} \\
+\left(\frac{b_{2}-2 c_{2} \mu_{2}}{\theta^{2}}+\frac{2 c_{2}}{\theta^{3}}\right) e^{\theta \mu_{2}}+\left(\frac{2 c_{1}}{\theta^{3}}-\frac{b_{1}+2 c_{1} \mu_{1}}{\theta^{2}}\right) e^{\theta \mu_{1}}
\end{gathered}
$$

The total amount of items which is perish within the time interval $\left[0, t_{1}\right]$ is 


$$
\begin{aligned}
D_{T}= & S-\int_{0}^{t_{1}} R(t) d t=S-\int_{0}^{\mu_{1}}\left(a_{1}+b_{1} t+c_{1} t^{2}\right) d t-\int_{\mu_{1}}^{\mu_{2}} R_{0} d t-\int_{\mu_{2}}^{t_{1}}\left(a_{2}+b_{2} t-c_{2} t^{2}\right) d t \\
& -\frac{a_{1}}{\theta}+\frac{b_{1}}{\theta^{2}}-\frac{2 c_{1}}{\theta^{3}}+\left(\frac{a_{2}+b_{2} t_{1}-c_{2} t^{2}}{\theta}-\frac{b_{2}-2 c_{2} t_{1}}{\theta^{2}}-\frac{2 c_{2}}{\theta^{3}}\right) e^{\theta t_{1}} \\
& +\left(-\frac{b_{1}+2 c_{1} \mu_{1}}{\theta^{2}}+\frac{2 c_{1}}{\theta^{3}}\right) e^{\theta \mu_{1}}+\left(\frac{b_{2}-2 c_{2} \mu_{2}}{\theta^{2}}+\frac{2 c_{2}}{\theta^{3}}\right) e^{\theta \mu_{2}}-a_{1} \mu_{1}-\frac{b_{1}}{2} \mu_{1}^{2}-\frac{c_{1}}{3} \mu_{1}^{3} \\
& -R_{0}\left(\mu_{2}-\mu_{1}\right)-a_{2}\left(t_{1}-\mu_{2}\right)-\frac{b_{2}}{2}\left(t_{1}^{2}-\mu_{2}^{2}\right)+\frac{c_{2}}{3}\left(t_{1}^{3}-\mu_{2}^{3}\right)
\end{aligned}
$$

The total amount of inventory carried during the time interval $\left[0, t_{1}\right]$ is

$$
\begin{aligned}
& C_{T}=\int_{0}^{t_{1}} I(t) d t \\
& =\int_{0}^{\mu_{1}} I(t) d t+\int_{\mu_{1}}^{\mu_{2}} I(t) d t+\int_{\mu_{2}}^{t_{1}} I(t) d t \\
& =\int_{0}^{\mu_{1}}\left[\begin{array}{l}
\frac{b_{1}+2 c_{1} t}{\theta^{2}}-\frac{2 c_{1}}{\theta^{3}}-\frac{a_{1}+b_{1} t+c_{1} t^{2}}{\theta}+\left(\frac{a_{2}+b_{2} t_{1}-c_{2} t_{1}^{2}}{\theta}-\frac{b_{2}}{\theta^{2}}+\frac{2 c_{2} t_{1}}{\theta^{2}}-\frac{2 c_{2}}{\theta^{3}}\right) e^{\theta\left(t_{1}-t\right)} \\
+\left(\frac{b_{2}-2 c_{2} \mu_{2}}{\theta^{2}}+\frac{2 c_{2}}{\theta^{3}}\right) e^{\theta\left(\mu_{2}-t\right)}+\left(\frac{2 c_{1}}{\theta^{3}}-\frac{b_{1}+2 c_{1} \mu_{1}}{\theta^{2}}\right) e^{\theta\left(\mu_{1}-t\right)}
\end{array}\right] d t \\
& +\int_{\mu_{1}}^{\mu_{2}}\left[\begin{array}{l}
-\frac{R_{0}}{\theta}+\left(\frac{a_{2}+b_{2} t_{1}-c_{2} t_{1}^{2}}{\theta}-\frac{b_{2}}{\theta^{2}}+\frac{2 c_{2} t_{1}}{\theta^{2}}-\frac{2 c_{2}}{\theta^{3}}\right) e^{\theta\left(t_{1}-t\right)} \\
+\left(\frac{b_{2}-2 c_{2} \mu_{2}}{\theta^{2}}+\frac{2 c_{2}}{\theta^{3}}\right) e^{\theta\left(\mu_{2}-t\right)}
\end{array}\right] d t \\
& +\int_{\mu_{2}}^{t_{2}}\left[\left(\frac{a_{2}+b_{2} t_{1}-c_{2} t_{1}^{2}}{\theta}-\frac{b_{2}}{\theta^{2}}+\frac{2 c_{2} t_{1}}{\theta^{2}}-\frac{2 c_{2}}{\theta^{3}}\right) e^{\theta\left(t_{1}-t\right)}+\frac{b_{2}-2 c_{2} t}{\theta^{2}}+\frac{2 c_{2}}{\theta^{3}}-\frac{a_{2}+b_{2} t-c_{2} t^{2}}{\theta}\right] d t \\
& =\left(\frac{a_{2}+b_{2} t_{1}-c_{2} t_{1}^{2}}{\theta}+\frac{2 c_{2} t_{1}-b_{2}}{\theta^{2}}-\frac{2 c_{2}}{\theta^{3}}\right)\left(\frac{e^{\theta t_{1}}-1}{\theta}\right)+\left(\frac{b_{2}-2 c_{2} \mu_{2}}{\theta^{2}}-\frac{2 c_{2}}{\theta^{3}}\right)\left(\frac{e^{\theta \mu_{2}}-1}{\theta}\right) \\
& +\left(\frac{2 c_{1}}{\theta^{3}}-\frac{b_{1}+2 c_{1} \mu_{1}}{\theta^{2}}\right)\left(\frac{e^{\theta \mu_{1}}-1}{\theta}\right)-\frac{a_{1} \mu_{1}}{\theta}-\frac{b_{1} \mu_{1}^{2}}{2 \theta}-\frac{c_{1} \mu_{1}^{3}}{3 \theta}+\left(\frac{b_{1}}{\theta^{2}}-\frac{2 c_{1}}{\theta^{3}}\right) \mu_{1}+\frac{c_{1} \mu_{1}^{2}}{\theta^{2}} \\
& -\frac{R_{0}}{\theta}\left(\mu_{2}-\mu_{1}\right)-\frac{a_{2}}{\theta}\left(t_{1}-\mu_{2}\right)-\frac{b_{2}}{2 \theta}\left(t_{1}^{2}-\mu_{2}^{2}\right)+\frac{c_{2}}{3 \theta}\left(t_{1}^{3}-\mu_{2}^{3}\right) \\
& +\left(\frac{b_{2}}{\theta^{2}}-\frac{2 c_{2}}{\theta^{3}}\right)\left(t_{1}-\mu_{2}\right)-\frac{c_{2}}{\theta^{2}}\left(t_{1}^{2}-\mu_{2}^{2}\right)
\end{aligned}
$$

Total quantity of shortage during the time interval $\left[t_{1}, T\right]$ is

$$
\begin{aligned}
B_{T} & =-\int_{t_{1}}^{T} I(t) d t=-\int_{t_{1}}^{T}\left[a_{2}\left(t_{1}-t\right)+\frac{b_{2}}{2}\left(t_{1}^{2}-t^{2}\right)+\frac{c_{2}}{3}\left(t^{3}-t_{1}^{3}\right)\right] d t \\
& =-a_{2} t_{1}\left(T-t_{1}\right)+\frac{a_{2}}{2}\left(T^{2}-t_{1}^{2}\right)-\frac{b_{2}}{2} t_{1}^{2}\left(T-t_{1}\right)+\frac{b_{2}}{6}\left(T^{3}-t_{1}^{3}\right)-\frac{c_{2}}{12}\left(T^{4}-t_{1}^{4}\right)+\frac{c_{2} t_{1}^{3}}{3}\left(T-t_{1}\right)
\end{aligned}
$$

Then, the total average cost per unit time under the time interval $\mu_{2} \leq t_{1} \leq T$, can be written as 


$$
A_{3}\left(t_{1}\right)=\frac{1}{T}\left[k_{0}+k_{1} D_{T}+k_{2} C_{T}+k_{3} B_{T}\right]
$$

The first order derivative of $A_{3}\left(t_{1}\right)$ with respect to $t_{1}$ is as follows:

$$
\frac{d A_{3}\left(t_{1}\right)}{d t_{1}}=\frac{1}{T}\left[\left(k_{1}+\frac{k_{2}}{\theta}\right)\left(e^{\theta t_{1}}-1\right)+k_{3}\left(t_{1}-T\right)\right]\left(a_{2}+b_{2} t_{1}-c_{2} t_{1}^{2}\right)
$$

The required necessary condition for $A_{3}\left(t_{1}\right)$ to be minimized is

$$
\begin{aligned}
& \frac{d A_{3}\left(t_{1}\right)}{d t_{1}}=0, \text { that is } \\
& \frac{1}{T}\left[\left(k_{1}+\frac{k_{2}}{\theta}\right)\left(e^{\theta_{1}}-1\right)+k_{3}\left(t_{1}-T\right)\right]\left(a_{1}+b_{1} t_{1}+c_{1} t_{1}^{2}\right)=0
\end{aligned}
$$

This implies that

$$
\begin{aligned}
& {\left[\left(k_{1}+\frac{k_{2}}{\theta}\right)\left(e^{\theta_{1}}-1\right)+k_{3}\left(t_{1}-T\right)\right]=0} \\
& \text { Let } p\left(t_{1}\right)=\left[\left(k_{1}+\frac{k_{2}}{\theta}\right)\left(e^{\theta_{1}}-1\right)+k_{3}\left(t_{1}-T\right)\right],
\end{aligned}
$$

since $p^{\prime}\left(t_{1}\right)=\left(k_{1}+\frac{k_{1}}{\theta}\right) e^{a_{1}} \theta+k_{3}>0$, which implies that $p\left(t_{1}\right)$ is strictly monotonically increasing function within the interval $t_{1} \in\left[\mu_{2}, T\right]$.

\section{Property-3}

In this case, the inventory model under the condition $\mu_{2} \leq t_{1}<T, A_{3}\left(t_{1}\right)$ attains its minimum at $t_{1}=t_{1}^{*}$, where $p\left(t_{1}^{*}\right)=0$ if $\mu_{2}<t_{1}^{*}$. On the other hand, $A_{3}\left(t_{1}\right)$ attains its minimum at $t_{1}^{*}=\mu_{2}$ if $t_{1}^{*}<\mu_{2}$. Now, we can calculate the total back-order quantity at the end of the cycle is

$\Delta_{3}=a_{2}\left(T-t_{1}^{*}\right)+\frac{b_{2}}{2}\left(T^{2}-t_{1}^{* 2}\right)+\frac{c_{2}}{3}\left(t_{1}^{* 3}-T^{3}\right)$.

Therefore, the optimal order quantity, denoted by $Q^{*}$, is $Q^{*}=S^{*}+\Delta_{3}$, where $S^{*}$ denotes the optimal value of $S$. From the above three cases, we can derive the following results

\section{Result-1}

An inventory model having constant deteriorating rate with quadratic trapezoidal type demand, the optimal replenishment time is $t_{1}^{*}$ and $A_{1}\left(t_{1}\right)$ attains its minimum at $t_{1}=t_{1}^{*}$ if and only if $t_{1}^{*}<\mu_{1}$. On the other hand, $A_{2}\left(t_{1}\right)$ attains its minimum at $t_{1}=t_{1}^{*}$ if and only if $\mu_{1}<t_{1}^{*}<\mu_{2}$ and $A_{3}\left(t_{1}\right)$ attains its minimum at $t_{1}=t_{1}^{*}$ if and only if $\mu_{2}<t_{1}^{*}$, where $t_{1}^{*}$ is the unique solution of equation $p\left(t_{1}\right)=0$.

\section{Example}

We can consider suitable values of the following parameters as follows: $T=12$ weeks, $\mu_{1}=4$ weeks, $\mu_{2}$ $=10$ weeks, $a_{1}=100$ unit, $b_{1}=5$ unit, $c_{1}=4$ unit, $a_{2}=220$ unit, $b_{2}=10$ unit, $c_{2}=2$ unit, $\theta=0.1, k_{0}=\$ 200$, $k_{1}=\$ 3$ per unit, $k_{2}=\$ 10$ per unit, $k_{3}=\$ 4$ per unit. Using the above data, we can find $p\left(\mu_{1}\right)=98.0951>0$, 
the optimal replenishment time $t_{1}^{*}=4.397$ weeks, the optimal order quantity $Q^{*}$, for each ordering cycle, is 4422.3465 unit and the minimum cost $A_{1}\left(t_{1}^{*}\right)=\$ 5848.1098$

Table 1

Sensitivity analysis

\begin{tabular}{|c|c|c|c|c|c|c|c|c|c|}
\hline Paramete & $(\%)$ & $\mathrm{t}^{*}{ }_{1}$ & $Q^{*}$ & $\mathrm{~A}_{1}\left(\mathrm{t}_{1}{ }^{*}\right)$ & Paramete & $(\%)$ & $\mathrm{t}^{*}{ }_{1}$ & $\mathrm{Q}^{*}$ & $\mathrm{~A}_{1}\left(\mathrm{t}_{1}{ }^{*}\right)$ \\
\hline \multirow{8}{*}{$a_{1}$} & +50 & 4.82 & 4688.507 & 5546.2 & \multirow{8}{*}{$\mathrm{C}_{2}$} & +50 & 4.637 & 4547.5 & 5753.0 \\
\hline & +25 & 4.71 & 4616.024 & 5538.8 & & +25 & 4.592 & 4498.3 & 5742.4 \\
\hline & +20 & 4.67 & 4582.499 & 5536.2 & & +20 & 4.581 & 4485.6 & 5740.2 \\
\hline & +10 & 4.61 & 4536.084 & 5534.3 & & +10 & 4.558 & 4459.2 & 5735.8 \\
\hline & -10 & 4.43 & 4448.777 & 5527.3 & & -10 & 4.522 & 4426.4 & 5725.7 \\
\hline & -20 & 4.28 & 4188.366 & 5523.2 & & -20 & 4.486 & 4403.5 & 5722.1 \\
\hline & -25 & 4.13 & 4117.554 & 5514.7 & & -25 & 4.471 & 4391.4 & 5719.6 \\
\hline & -50 & 3.99 & 3968.533 & 5541.4 & & -50 & 4.393 & 4333.1 & 5707.0 \\
\hline \multirow{8}{*}{$\mathrm{b}_{1}$} & +50 & 4.53 & 4549.407 & 5646.0 & \multirow{8}{*}{$\Theta$} & +50 & 4.748 & 4573.6 & 5865.2 \\
\hline & +25 & 4.5 & 4523.039 & 5634.9 & & +25 & 4.688 & 4495.6 & 5857.8 \\
\hline & +20 & 4.47 & 4474.111 & 5530.1 & & +20 & 4.566 & 4473.4 & 5851.3 \\
\hline & +10 & 4.42 & 4436.456 & 5525.3 & & +10 & 4.51 & 4422.3 & 5848.1 \\
\hline & -10 & 4.3 & 440.8903 & 5519.7 & & -10 & 4.487 & 4379.8 & 5841.6 \\
\hline & -20 & 4.28 & 4385.378 & 5510.3 & & -20 & 4.417 & 4366.9 & 5835.3 \\
\hline & -25 & 4.21 & 4325.333 & 5499.8 & & -25 & 4.34 & 4359.8 & 5830.6 \\
\hline & -50 & 4.18 & 4300.178 & 5487.3 & & -50 & 4.319 & 4344.5 & 5823.1 \\
\hline \multirow{8}{*}{$\mathrm{c}_{1}$} & +50 & 4.05 & 4665.328 & 5230.3 & \multirow{8}{*}{$k_{1}$} & +50 & 3.57 & 3725.3 & 4955.6 \\
\hline & +25 & 4.29 & 4223.772 & 4886.8 & & +25 & 4.076 & 4057.7 & 4703.2 \\
\hline & +20 & 4.33 & 3943.030 & 4815.8 & & +20 & 4.294 & 4152.7 & 4677.2 \\
\hline & +10 & 4.42 & 3689.353 & 4675.3 & & +10 & 4.375 & 4326.9 & 4605.4 \\
\hline & -10 & 4.74 & 3187.698 & 4387.0 & & -10 & 4.552 & 4560.3 & 4465.4 \\
\hline & -20 & 4.81 & 2931.327 & 4342.3 & & -20 & 4.664 & 4648.0 & 4397.6 \\
\hline & -25 & 5.27 & 2693.037 & 4367.8 & & -25 & 4.854 & 4702.0 & 4302.0 \\
\hline & -50 & 5.42 & 2114.598 & 4295.3 & & -50 & 4.908 & 4798.8 & 4195.0 \\
\hline \multirow{8}{*}{$\mathrm{a}_{2}$} & +50 & 4.94 & 4877.508 & 5916.9 & \multirow{8}{*}{$k_{2}$} & +50 & 3.927 & 3744.7 & 4675.3 \\
\hline & +25 & 4.91 & 4803.015 & 5876.1 & & +25 & 3.832 & 3678.8 & 4614.2 \\
\hline & +20 & 4.88 & 4771.499 & 5842.5 & & +20 & 3.723 & 3635.4 & 4600.7 \\
\hline & +10 & 4.81 & 4726.773 & 5811.2 & & +10 & 3.687 & 3548.8 & 4569.2 \\
\hline & -10 & 4.76 & 4710.000 & 5786.9 & & -10 & 3.613 & 3333.0 & 4485.1 \\
\hline & -20 & 4.68 & 4694.886 & 5774.5 & & -20 & 3.456 & 3186.2 & 4427.3 \\
\hline & -25 & 4.53 & 4617.663 & 5737.7 & & -25 & 3.341 & 3106.5 & 4392.8 \\
\hline & -50 & 4.47 & 4579.003 & 5689.1 & & -50 & 3.28 & 2537.8 & 4113.9 \\
\hline \multirow{8}{*}{$\mathrm{b}_{2}$} & 50 & 3.88 & 3778.544 & 5745.6 & \multirow{8}{*}{$k_{3}$} & 50 & 4.267 & 3823.4 & 4437.6 \\
\hline & 25 & 4.38 & 4230.436 & 5682.3 & & 25 & 3.883 & 3752.9 & 4485.3 \\
\hline & 20 & 4.44 & 4278.083 & 5622.8 & & 20 & 3.826 & 3658.2 & 4492.0 \\
\hline & 10 & 4.54 & 4375.889 & 5577.7 & & 10 & 3.652 & 3630.9 & 4512.7 \\
\hline & -10 & 4.62 & 4530.839 & 5484.8 & & -10 & 3.056 & 3577.4 & 4544.3 \\
\hline & -20 & 4.69 & 4580.458 & 5437.9 & & -20 & 2.866 & 3109.2 & 4564.3 \\
\hline & -25 & 4.73 & 4625.320 & 5412.2 & & -25 & 2.544 & 3014.6 & 4604.9 \\
\hline & -50 & 4.99 & 4673.683 & 5392.3 & & -50 & 1.987 & 2995.1 & 4687.3 \\
\hline
\end{tabular}

In the above table some sensitivity analysis of the model is performed by changing the parameter $-50 \%$, $-25 \%,-20 \%,-10 \%,+10 \%,+20 \%,+25 \%$ and $+50 \%$ taking one at a time and keeping the remaining unchanged. 


\section{Conclusion}

In a realistic product life cycle, demand is increasing with time during the growth phase. Then, after reaching its peak, the demand becomes stable for a finite time period called the maturity phase. Thereafter, the demand starts decreasing with time. Therefore, in this paper, we have studied the inventory model for constant deteriorating items with quadratic trapezoidal demand rate. We have proposed an inventory replenishment policy for this type of inventory model. From the market information, we have found that the quadratic trapezoidal type demand rate was more realistic than ramp type demand rate, constant demand rate and other time dependent demand rate Our paper provides an interesting topic for the future study of such kind of important inventory models, and at the same time, the following problems can be considered for future research work (1) How about the inventory model starting with shortages? (2) How about the inventory model with time dependent deteriorating rate instead of constant deteriorating rate?

\section{Acknowledgement}

The authors are very much thank full to anonymous referee for his valuable comments, which has improved the work. His suggestions also opened new avenues for young researchers.

\section{References}

Abad, P. L. (1996). Optimal pricing and lot sizing under conditions of perish-ability and partial back ordering. Management Science, 42, 1093-1104.

Abad, P. L. (2001). Optimal price and order size for a reseller under partial back ordering. Computers and Operations Research, 28, 53-65.

Benkherouf, L., Boumenir, A., \& Aggoun, L. (2003). A diffusion inventory model for deteriorating items. Applied Mathematics and Computation, 138, 21-39.

Bhattacharya, D. K. (2005). On multi item inventory. European Journal of Operational Research, 162, 786-791.

Cheng, M., Zhang, B., \& Wang, G. (2011). Optimal policy for deteriorating items with trapezoidal type demand and partial backlogging. Applied Mathematical Modeling, 35, 3552-3560.

Chu, P., Yang, K. L., Liang, S. K., \& Niu, T. (2004). Note on inventory model with a mixture of back orders and lost sales. European Journal of Operational Research, 159, 470-475.

Chung, K. J., Chu, P., \& Lan, S. P. (2000). A note on EOQ models for deteriorating items under stock dependent selling rate. European Journal of Operational Research, 124, 550-559.

Chung, K. J., \& Lin, C. N. (2001). Optimal inventory replenishment models for deteriorating items taking account of time discounting. Computers and Operations Research, 28, 67-83.

Chung, M., \& Wang, G. (2009). A note on the inventory model for deteriorating items with trapezoidal type demand rate. Computers and Industrial Engineering, 56, 1296-1300.

Dave, U. (1989). A deterministic lot size inventory model with shortages and a linear trend in demand. Naval Research Logistics, 36, 507-514.

Deng, P. S. (2005). Improved inventory models with ramp type demand and Weibull deterioration. International Journal of Information and Management Sciences, 16, 79-86.

Deng, P. S., Lin, R., \& Peter, Chu. P. (2007). A note on inventory models for deteriorating items with ramp type demand rate. European Journal of Operational Research, 178, 112-120.

Ghare, P. M., \& Schrader, G. P. (1963). A model for an exponentially decaying inventory, Journal of Industrial Engineering, 14, 5.

Giri, B. C., \& Chaudhuri, K. S. (1998). Deterministic models of perishable inventory with stock dependent demand rate and non-linear holding cost. European Journal of Operational Research, 105, 467-474. 
Giri, B. C., Jalan, A. K., \& Chaudhuri, K. S. (2003). Economic order quantity model with Weibull deterioration distribution, shortage and ramp type demand, International Journal of system and science, 34, 237-243.

Hennery, R. J. (1979). Inventory replenishment policy for increasing demand. Journal of the Operational Research Society, 30, 611-617.

Hill, R. M. (1995). Inventory models for increasing demand followed by level demand. Journal of the Operational Research Society, 46, 1250-1259.

Hung, K. C. (2011). An inventory model with generalized type demand, deterioration and back order rates. European Journal of Operational Research, 208, 239-242.

Mandal, B., \& Pal, A. K. (1998). Order level inventory system with ramp type demand rate for deteriorating items. Journal of Interdisciplinary Mathematics, 1, 49-66.

Manna, S. K., \& Chaudhuri, K. S. (2006). An EOQ model with ramp type demand rate, time dependent deterioration rate, unit production cost and shortages. European Journal of Operational Research, 171, 557-566.

Mishra, V. K., Singh, L. S., \& Kumar, R. (2013). An inventory model for deteriorating items with time dependent demand and time varying holding cost under partial backlogging. Journal of Industrial Engineering International, 9, 4-8.

Padmanabhan, G. (1990). Inventory model with a mixture of back-orders and lost sales. International Journal of System Science, 21, 1721-1726.

Panda, G. C., Sahoo, S., \& Sukla, P. K. (2012), Analysis of constant deteriorating inventory management with quadratic demand rate. American Journal of Operational Research, 2, 98-103.

Panda, S., Senapati, S., \& Basu, M. (2008). Optimal replenishment policy for perishable seasonal products in a season with ramp type time dependent demand. Computers and Industrial Engineering, 54, 301-314.

Panda, S., Saha, S., \& Basu, M. (2007). An EOQ model with generalized ramp type demand and Weibull distribution deterioration. Asia-Pacific Journal of Operations Research, 24, 93-109.

Resh, M., Friedman, M., \& Barbosa, L. C. (1979). On a general solution of the deterministic lot size problem with time proportional demand. Operations Research, 24, 718-725.

Sachan, R. S. (1984). On policy inventory model for deteriorating items with time proportional demand. Journal of the Operational Research Society, 35, 1013-1019.

Set, B. K., Sarkar, B., \& Goswami, A. (2013). A two-warehouse inventory model with increasing demand and time varying deterioration. Scientia Iranica E, 19, 1969-1977.

Teng, J. T. (1996). A deterministic replenishment model with linear trend in demand. Operations Research Letters, 19, 33-41.

Teng, J. T., Chang, H. J., Dye, C. Y., \& Hung, C. H. (2002). An optimal replenishment policy for deteriorating items with time varying demand and partial backlogging. Operations Research Letters, 30, 387-393.

Wee, H. M. (1993). Economic production lot size model for deteriorating items with partial back ordering. Computer and Industrial Engineering, 24, 449-458.

Wee, H. M. (1995). Joint price and replenishment policy for deteriorating inventory with declining market. International Journal of Production Economics, 40, 163-171.

Wee, H. M., \& Law, S. T. (1999). Economic production lot size for deteriorating items taking account of the time value of money. Computers and Operations Research, 26, 545-558.

Whitin, T. M. (1957). Theory of inventory management. Princeton University Press, Princeton NJ, 6272.

Wu, J. W., Lin, C., Tan, B., \& Lee, W. C. (1999). An EOQ with ramp type demand rate for deteriorating items with Weibull deterioration. International Journal of Information and Management Sciences, 10, 41-51.

Wu, K. S. (2001). An EOQ inventory model for items with Weibull distribution deterioration, ramp type demand rate and partial back logging. Production Planning and Control, 12, 787-793.

Wu, K. S., \& Ouyang, L. Y. (2000). A replenishment policy for deteriorating items with ramp type demand rate. Proceeding of National Science Council ROC (A), 24, 279-286. 
Wu, K. S., Ouyang, L. Y., \& Yang, C. T. (2006). An optimal replenishment policy for non-instantaneous deteriorating items with stock dependent demand and partial back logging. International Journal of Production Economics, 101, 369-384.

Wu, K. S., Ouyang, L. Y., \& Yang, C. T. (2008). Retailer's optimal ordering policy for deteriorating items with ramp type demand under stock dependent consumption rate. International Journal of Information and Management Sciences, 19, 245-262. 